\title{
ATENÇÃO A PESSOAS DEPENDENTES DE CRACK EM UM CENTRO DE ATENÇÃO PSICOSSOCIAL
}

\section{ATTENTION PEOPLE CRACK OF DEPENDENT ON A PSYCHOSOCIAL CARE CENTER}

\author{
Maria de Lourdes Custódio Duarte, ${ }^{1}$ Kelly Viana, ${ }^{1}$ Agnes Olschowsky ${ }^{1}$ \\ ${ }^{1}$ Universidade Federal do Rio Grande do Sul-UFRGS/Porto Alegre/Brasil. \\ Autor correspondente: Maria de Lourdes Custódio Duarte e-mail: malulcd@ yahoo.com.br
}

\section{EDITORES}

Thiago Gomes Heck

(Unijuí-Brasil)

Adriane Cristina Bernat Kolankiewicz

(Unijuí-Brasil)

\section{EDITORES DE ÁREA}

Educação \& Saúde

Eva Teresinha de Oliveira Boff

(Unijuí-Brasil)

Fisioterapia \& Saúde

Eliane Roseli Winkelmann

(Unijuí-Brasil)

Ciências Farmacêuticas \& Saúde

Marilei Uecker Pletsch

(Unijuí-Brasil)

\section{Nutrição \& Saúde}

Lígia Beatriz Bento Franz

(Unijuí-Brasil)

Nadia Oliveira

(Unipampa-Brasil)

Ingrid Perry

(UNESC-Brasil)

Enfermagem e suas contribuições para a prática

Adriane Cristina Kolankiewicz

(Unijuí-Brasil)

Crhis de Brum

(UFFS-Brasil)

Neila de Souza

(UFSM-Brasil)

\section{Exercício Físico \& Saúde}

Thiago Gomes Heck

(Unijuí-Brasil)

Anderson Zampier Ulbrich

Editora Unijuí

Universidade Regional do Noroeste do Estado do Rio Grande do Sul (Unijuí)

\section{RESUMO}

A atenção aos usuários de crack vem sendo garantida a partir das reformulações da legislação brasileira, tendo em vista que o modelo tradicional e excludente de assistência psiquiátrica modificou-se a partir da Reforma Psiquiátrica. Assim, os Centros de Atenção Psicossocial (Caps) Álcool e Drogas se constituem em serviços criados a partir da reforma psiquiátrica que têm como propósito a atenção em saúde mental no âmbito do território. Tem-se por objetivo relatar as estratégias utilizadas por um Caps AD da fronteira oeste do Rio Grande do Sul para a garantia da atenção aos usuários de crack. Este estudo trata-se de um relato de experiência e, para isso, observou-se o cotidiano do serviço do período de julho a outubro de 2012. Evidenciou-se que os grupos de apoio, as oficinas profissionalizantes e as visitas domiciliares realizadas por esse serviço são entendidas como atividades estratégicas para o cuidado integral e a garantia da atenção aos usuários de crack por um atendimento de qualidade, na lógica do Sistema Único de Saúde.

Palavras-chave: Saúde mental. Transtornos relacionados ao uso de substâncias. Atenção à Saúde. Serviços comunitários de saúde mental.

Submetido em: $17 / 12 / 2015$

Aceito em: 23/10/2016 


\section{Revista}

\section{Contexto}

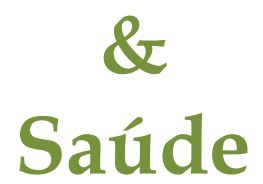

Volume 16

Número 31

2016

ISSN 2176-7114

\begin{abstract}
Attention to crack users has been guaranteed from reformulations Brazilian law, given that the traditional and exclusionary model of psychiatric care has changed from the Psychiatric Reform. Thus, the Centers for Psychosocial Care (CAPS) Alcohol and Drugs constitute services created from the psychiatric reform that aims to mental health care in the territory. It has the objective to describe the strategies used by a CAPS AD the western border of Rio Grande do Sul to guarantee the attention to crack users. This study it is an experience report and that there was the daily service from July to October 2012. It was evident that the Support Groups, the Vocational workshops and home visits by this service are understood as activities strategies for comprehensive care and ensuring attention to crack users a quality service, the logic of the Unified Health System.
\end{abstract}

Keywords: Mental health. Disorders related to substance use. Health Care. Community mental health services.

\begin{abstract}
A Revista Contexto \& Saúde é um periódico do Departamento de Ciências da Vida da Universidade Regional do Noroeste do Estado do Rio Grande do Sul (Unijuí). É um periódico semestral que tem por objetivo a divulgação da produção técnico-científica de temas relacionados à área de Ciências da Saúde.

O escopo da revista abrange a divulgação de resultados de pesquisa que contemplem avanços no processo saúde-doençacuidado e no conhecimento e aplicabilidade de novos processos químicos e biológicos em saúde.

Neste periódico, entende-se que a publicação de estudos com os aspectos epidemiológicos, assistenciais e educacionais em saúde, experimentais e aplicados é uma forma a subsidiar e qualificar a atenção à saúde de modo interdisciplinar.
\end{abstract}




\section{INTRODUÇÃO}

A legislação brasileira de saúde vem sofrendo significativas transformações, principalmente no que se refere à saúde mental, tendo em vista que o modelo tradicional e excludente de assistência psiquiátrica, o modelo asilar, modificou-se a partir do movimento intitulado Reforma Psiquiátrica. Este movimento, de âmbito mundial, repercutiu no Brasil nos últimos anos, mais precisamente no final de década de 70, tendo como um dos objetivos a atenção às pessoas com sofrimento mental, seja ele em decorrência ou não de dependência química.

A lei 10.216/2001, que instituiu a Reforma Psiquiátrica no Brasil, enfatiza a cidadania em contraposição à antiga lógica, excludente e estigmatizante, das políticas de saúde voltadas ao atendimento do "doente mental" e do "viciado". Essa lei destaca-se por reafirmar o direito do usuário à convivência sociofamiliar, ao mesmo tempo em que contempla as singularidades possíveis dos tratamentos. As ações de cuidado são amplas e devem ser consideradas caso a caso, sem preconceitos de qualquer espécie (BRASIL, 2001).

A Atenção Psicossocial, preconizada pela Reforma Psiquiátrica, tem se mostrado como um paradigma desencadeador de tensões na operacionalização das práticas nos serviços de saúde. É justamente neste campo de tensão entre o novo e o velho, do conflito entre a atenção psicossocial e o modelo psiquiátrico tradicional, contudo, que surge a possibilidade de ruptura da ideologia do tradicional (AZEVEDO; MIRANDA, 2010). O modelo tradicional e asilar visa apenas à remissão dos sintomas, à desconsideração do sujeito como cidadão, levando-o à segregação, tutela e isolamento social, violando os direitos das pessoas em sofrimento.

Associado à cidadania, à integralidade e à intersetorialidade, o modo psicossocial busca a reabilitação psicossocial do sujeito, por meio da reconstrução do cotidiano da pessoa com diagnóstico de transtorno mental (VARELA et al., 2016). Essa reconstrução é determinada pela capacidade de lidar com problemas cotidianos, pelo desenvolvimento da autoestima, pelas habilidades sociais, autonomia e prática da cidadania.

Concorda-se com Azevedo e Miranda (2010) quando afirmam que a luta por uma assistência psiquiátrica mais humanizada vai ao encontro da garantia dos direitos sociais dos sujeitos enquanto cidadãos sejam eles pessoas com transtornos mentais ou dependentes químicos.

Nesse sentido, a partir dos anos 90, a literatura nacional e internacional traz um aumento do consumo de drogas no Brasil e no mundo, e, consequentemente, o elevado grau de dependência química que elas causam no usuário. Dentre elas destaca-se o crack. Assim, o consumo de crack emerge no cenário brasileiro no final da década de 80, apresentando-se como um fenômeno de rápida expansão, principalmente nos grandes centros urbanos (DUARTE, 2013).

Na cidade de São Paulo, em 1989, houve o primeiro relato do uso de crack no Brasil. Dois anos depois, a primeira apreensão policial da droga e, em 1993, ocorreram 204 registros de apreensões. Já em 1995 foram registrados 1.906 casos, evidenciando a rápida popularização de crack no país (OLIVEIRA; NAPPO, 2008).

O perfil dos usuários de crack brasileiros não difere muito daqueles encontrados em outros países. Eles caracterizam-se por serem jovens, desempregados, com baixa escolaridade, baixo poder aquisitivo, que apresentam antecedentes de uso de drogas e com comportamento de risco, sendo provenientes de famílias desestruturadas. O crack, no entanto, tem se tornado popular nos centros urbanos, atingindo também as áreas rurais (FERTIG et al, 2016). Crianças, moradores de rua e mulheres tornam-se vulneráveis à droga (CFM, 2011), além de atingir os adultos de diversos segmentos econômicos. 
Outra característica dos usuários de cocaína e crack é o policonsumo, ou seja, raramente essas substâncias são usadas de forma isolada, o que aumenta a exposição dos usuários a patologias associadas ao consumo dessas outras drogas, sejam elas ilícitas ou lícitas. O tabaco, o álcool e a maconha são algumas das substâncias usadas concomitantemente com o crack, o que possibilita o aumento de consequências adversas, maiores problemas psicossociais e tendência à incompatibilidade com os tratamentos. Dificuldades em receber visitas e aumento das recaídas somam-se às consequências do policonsumo de drogas desses usuários (BOYLE et al., 2010).

Outro aspecto importante enfatizado pelo Ministério da Saúde (Brasil, 2010b; Brasil, 2010c) diz respeito aos dispositivos da rede de atenção em saúde mental utilizados pelos usuários de crack e seus familiares para buscar ajuda, apoio, orientação e tratamento.

O cuidado deve ser organizado em uma rede horizontal de atenção à saúde, com todos os trabalhadores responsáveis pela gestão do cuidado articulados no sentido de atender às necessidades de cuidado expressas por pessoas. Para que isso seja possível e exequível, é necessário que a rede de atenção em saúde mental oferte outros pontos de cuidado para os usuários de crack. Esses pontos de cuidados podem ser: o Ambulatório de Saúde Mental, o Amor Exigente (AE), o Hospital Geral, a Fazenda Terapêutica, o Conselho Tutelar, a Promotoria, o Consultório na Rua, o Centro de Orientação e Apoio Sorológico (Coas), o Centro de Referência de Assistência Social (Cras) e o Centro de Referências Especializado em Assistência Social (Creas) entre outros (DUARTE, 2013).

Assim, a atenção em saúde mental a usuários de crack no âmbito do Sistema Único de Saúde (SUS) deverá pautar-se na atenção em rede, no acesso universal e na intersetorialidade.

No contexto de serviços da rede para o usuário de crack, destaca-se o Caps Álcool e Drogas (AD) III do município de Uruguaiana no Rio Grande do Sul. Segundo a Portaria no 2841 de 2010 que institui no âmbito do SUS o CAPS AD III, entende-se por aquele estabelecimento destinado a proporcionar atenção integral e contínua a pessoas com transtornos decorrentes do uso abusivo e da dependência de álcool e outras drogas, com funcionamento durante 24 horas por dia, inclusive nos feriados e finais de semana (Brasil, 2002).

Este estudo é um relato de experiência de discentes do curso de Enfermagem da Universidade Federal do Pampa (Unipampa) em um estágio no Caps AD III de Uruguaiana, no período de julho a outubro de 2012 sobre as estratégias utilizadas por esse serviço para garantir os direitos humanos dos usuários de crack.

Tem-se, portanto, por objetivo, relatar as estratégias utilizadas pelo serviço para a garantia dos direitos humanos de usuários de crack. Acredita-se que este estudo oportunizará dar visibilidade à atenção aos usuários de crack, propiciando mudanças e consolidando o cuidado integral a eles, na garantia dos seus direitos humanos.

Dessa forma, vislumbra-se que este estudo possa contribuir na construção do conhecimento sobre a área de Saúde Mental e Dependência Química, além de possibilitar reflexões sobre as estratégias utilizadas pelo Caps AD III para garantir a atenção aos usuários de crack. Assim, esta pesquisa pode configurar-se em um subsídio valioso para repensar a organização desses serviços na rede de atenção dos serviços de saúde mental, sendo justificada na relevância do tema na perspectiva do cuidado integral e dos pressupostos da Reforma Psiquiátrica.

\section{METODOLOGIA}

Trata-se de um estudo qualitativo (Minayo, 2013), do tipo relato de experiência realizado em um Caps AD III do município de Uruguaiana, localizado na fronteira Oeste do Rio Grande do Sul. Sua área é de $5.713 \mathrm{~km}^{2}$, possuindo uma população de 123.743 habitantes, sendo $93 \%$ concentrados na zona urbana (INSTITUTO..., 2009). 
Uruguaiana limita-se com o Uruguai e a Argentina, sendo porta de entrada do Mercado Comum do Sul (Mercosul), responsável por um grande volume de entrada e saída de caminhões vindo de várias partes da América do Sul e possuidor do maior porto seco da América Latina, o que possibilita a entrada de substâncias ilegais no município, gerando o aumento de apreensões de crack e de outras drogas (URUGUAIANA, 2010).

A cidade conta com serviços específicos na área de saúde mental, a saber: Ambulatório de Saúde Mental, leitos destinados à internação psiquiátrica no Hospital Geral da Santa Casa, o Centro de Atenção Psicossocial II Asas da Liberdade e o Caps AD III Homero Tarragô. Há, no entanto, outros serviços que compõem a rede, como o Centro de Atendimento a Crianças e Adolescentes (Cacau), Fundação de Atendimento Socioeducativo (Fase), Grupo de Trabalho Amor Especial (GTAE) e a Atenção Básica por meio da ESF (DUARTE, 2013).

O Caps AD III Homero Tarragô, foco deste estudo, foi resultado de reivindicações dos gestores de saúde mental do município de Uruguaiana, juntamente com a população, pela criação de um serviço especializado que atendesse à grande demanda de dependentes químicos, em especial usuários de crack (URUGUAIANA, 2010; DUARTE, 2013). Dessa forma, após diversas negociações sobre onde esse serviço iria ser alocado, em 17 de julho de 2012 o Caps AD III Homero Tarragô entrou em funcionamento. O Caps AD III já tinha como premissa, desde a construção do seu Projeto, a proposta da garantia dos direitos humanos dos usuários de crack como um dos alicerces do tratamento da dependência química, além de propor sua inclusão social (URUGUAIANA, 2010).

Dentre as inúmeras características desse tipo de Caps, salienta-se as seguintes: a) constituir-se em um serviço aberto, de base comunitária, funcionando na lógica do território; b) responsabilizar-se pela organização da demanda e da rede de cuidados em saúde mental, álcool e outras drogas; c) possuir capacidade técnica para desempenhar o papel de regular e articular as ações de atenção integral aos usuários de crack, álcool e outras drogas (BRASIL, 2010c).

Atualmente o Caps AD III conta com uma equipe composta de 30 profissionais, a saber: quatro psicólogos, uma nutricionista, uma terapeuta ocupacional, seis cuidadores, um enfermeiro, uma secretária, uma assistente social, duas estagiárias de serviço social, um educador físico, uma fisioterapeuta, três oficineiras, um segurança, seis técnicos de enfermagem e uma pedagoga.

A área física do Caps AD III é constituída por um hall de entrada, três salas para oficinas, uma sala para a recepção, um posto de enfermagem, duas salas para atendimentos, cinco banheiros, uma cozinha, um refeitório, uma sala de almoxarifado, uma sala da coordenação, um ambiente de lavanderia, um pátio, cinco corredores, quatro salas sem uso no momento do estudo, duas escadas de acesso ao $2^{\circ}$ andar, uma sala destinada ao pessoal terceirizado da higienização e uma sala que, futuramente, será utilizada para a alocação dos leitos-sala observação.

Móveis novos, tapetes e cartazes confeccionados pelos usuários e detalhes coloridos em EVA foram estratégicos para criar um ambiente agradável, superando o ambiente pouco "acolhedor" de muitos serviços de saúde. Nesse sentido, torna-se imprescindível constituir espaços e modos de acolher o sofrimento que comportem as diferentes dimensões decorrentes do processo de adoecimento mental, superando a visão individualizada, sintomática e reducionista da crise (KANTORSKI et al., 2011). Dessa forma, a ambiência de um serviço como o Caps AD é de extrema importância e colabora para a garantia da atenção aos usuários de crack, diferente da lógica do manicômio, na qual a ambiência não era acolhedora e os espaços eram frios. Nesse sentido, evidencia-se que os conceitos de ambiência preconizados pela política de humanização (BRASIL, 2005) foram incorporados pelo Caps AD III Homero Tarragô. 
Na tentativa de superar a lógica manicomial na qual a violação dos direitos de dependentes químicos eram violados, bem como a falta de atenção a esses usuários, o Caps AD III Homero Tarragô destina uma série de atividades voltadas para os usuários de crack e outras drogas, tais como: acolhimento, tratamento medicamentoso, atendimento em grupos para usuários, atendimento individualizado aos usuários, orientações, atendimento psicoterápico, atividades de suporte social, atendimento domiciliar, oficinas terapêuticas, de alfabetização e visitas domiciliares.

\section{RESULTADOS}

\section{ESTRATÉGIAS PARA A ATENÇÃO AOS USUÁRIOS DE CRACK NO CAPS AD III}

A garantia da atenção aos usuários de crack no Caps AD III do município de Uruguaiana ocorre de inúmeras formas. Para fins deste estudo, abordar-se-á os Grupos de Apoio, Oficinas Profissionalizantes e as Visitas Domiciliares por considerar que essas atividades e ações desenvolvidas no Caps AD III são importantes estratégias para a garantia da atenção e do cuidado integral ao usuário de crack.

Os grupos de apoio têm apresentado uma demanda crescente no contexto nacional e internacional e vêm se consolidando como uma modalidade de cuidado eficaz nos Caps (KEMPER et al., 2015) para os usuários de crack, álcool e outras substâncias psicoativas. Dessa forma, a terapia em grupo foi proposta primeiramente em razão do aumento da demanda de usuários no Caps AD III, no entanto os profissionais do serviço deram-se conta de que os grupos podem ser uma importante ferramenta de intervenção (RIBEIRO et al., 2012) e de apoio aos seus participantes.

Um grupo se caracteriza por todos que o integram estarem reunidos em tarefa e objetivo comuns (OLIVEIRA et al., 2010). No Caps AD III Homero Tarragô percebe-se que os usuários de crack utilizam-se do espaço do grupo como uma forma de desabafo, na qual o coordenador do grupo, percebendo essa necessidade, possibilitava que o protagonista desse espaço pudesse ser o usuário e não o profissional. Durante os grupos observados neste serviço, em diversos momentos, usuários choraram em seus depoimentos e o grupo, enquanto espaço terapêutico era continente dando resposta às necessidades de cuidados explícitas naquele momento. Além disso, os grupos de apoio empoderam os usuários com informações sobre os mais diversos assuntos, permitindo maior instrumentalização sobre sua dependência e informações sobre os efeitos do crack no seu organismo.

Nesse contexto também inserem-se as oficinas profissionalizantes. O Ministério da Saúde, pela Portaria No 189/91 (BRASIL, 1991), definiu as Oficinas como atividades grupais de socialização, expressão e inserção social. Assim, o termo oficina vem sendo empregado para designar atividades que permitem à pessoa promoção do exercício da cidadania e da expressão de liberdade ou um espaço de atividades manuais que possibilita a interação e a convivência entre as pessoas (NASCIMENTO; PÍTIA, 2010), na perspectiva da reinserção social e da atenção ao usuário de crack.

As oficinas profissionalizantes são importantes no contexto dos serviços substitutivos, como os Caps AD, porque proporcionam espaços de capacitação ao usuário-cidadão, auxiliando-lhe na reinserção na vida cotidiana. Oficinas de marcenaria, de padeiro e de mecânico foram ofertadas aos usuários de crack pelo serviço, possibilitando formação especializada e garantindo a oportunidade de reinserção no mercado de trabalho.

Por fim, outra estratégia utilizada pelo Caps AD III para garantir a atenção aos usuários de crack a um atendimento de qualidade e personalizado são as Visitas Domiciliares (VDs) (DUARTE; VIANA; OLSCHOWSKY, 2015). Durante a realização das VDs, usuários de crack sentem-se "resgatados" no sentido de estarem sendo assistidos no seu próprio domicílio pelo serviço de saúde responsável por aquela área de abrangência. A ação de sair de dentro do serviço denota uma postura ativa do profissional de ir ao encontro 
daqueles usuários que não acessam o Caps AD III ou, mesmo, de conhecer melhor a realidade daqueles que já participam do cotidiano do serviço.

Assim, a VD é um dispositivo de extrema importância na atenção ao usuário de crack utilizado pelo Caps AD III Homero Tarragô. O modelo psicossocial propõe que fatores políticos, biopsíquicos e socioculturais sejam tomados como determinantes das doenças. Dessa forma, as terapias sairiam do escopo medicamentoso exclusivo, ou preponderante, e o sujeito ganharia destaque como participante principal no tratamento, sendo a família e, eventualmente, um grupo mais ampliado, também incluídos como agentes fundamentais do cuidado (BRAGA; D’OLIVEIRA, 2015).

Os Grupos de Apoio, as Oficinas Profissionalizantes e as Visitas Domiciliares realizadas pelo Caps AD III do município de Uruguaiana, portanto, são entendidas como atividades estratégicas para a atenção integral aos usuários de crack por um atendimento de qualidade na lógica do Sistema Único de Saúde (SUS).

\section{CONSIDERAÇÕES FINAIS}

Os Caps AD configuram-se em serviços substitutivos à lógica do manicômio por entenderem o dependente químico, no caso deste estudo, usuário de crack, como pessoas que devem ser assistidas na perspectiva da atenção integral. $\mathrm{O}$ atendimento a esses usuários deve ser permeado pelas tecnologias relacionais como o acolhimento, a escuta qualificada e o vínculo. Essas tecnologias em saúde são potentes na lógica do cuidado dos serviços territoriais, como os Caps.

Nesse cenário, o Caps AD III do município de Uruguaiana utiliza três principais estratégias de atenção aos usuários de crack: os Grupos de Apoio, as Oficinas Profissionalizantes e as Visitas Domiciliares. Essas atividades configuram-se em dispositivos de extrema importância no contexto dos serviços substitutivos preconizados pela Reforma Psiquiátrica.

Salienta-se, portanto, que os Caps AD devem desenvolver atividades e ações na perspectiva da Reforma Psiquiátrica, oportunizando ao usuário de crack formas de reabilitação e de inclusão social, a fim de garantir a atenção e cuidado a essas pessoas que, por muito tempo, ficaram excluídas da sociedade.

\section{REFERÊNCIAS}

AZEVEDO, D. M.; MIRANDA, F. A. N. Práticas profissionais e tratamento ofertado nos CAPSad. Esc Anna Nery Rev Enferm., v. 14, n. 1, p. 56-63 jan./mar. 2010.

BRASIL. Ministério da Saúde. Portaria 189, de 19 de novembro 1991. Define necessidade de compatibilizar os procedimentos das ações de saúde mental com o modelo assistencial vigente. Brasília, DF, 1991.

BRAGA, C. P.; D'OLIVEIRA, A. F. P. L. The continuity of psychiatric hospitalization of children and adolescents within the Brazilian Psychiatric Reform scenario. Interface, Botucatu, v. 19, n. 52, p. 33-44, 2015.

BOYLE, M. J. et al. Attitudes of undergraduate health science students towards patients with intellectual disability, substance abuse, and acute mental illness: a cross-sectional study. BMC Med. Educ., London, v. 10, p. 71, oct. 2010.

BRASIL. Ministério da Saúde. Lei n. 10.216, de 6 de abril de 2001. Dispõe sobre a proteção e os direitos das pessoas portadoras de transtornos mentais e redireciona o modelo assistencial em saúde mental. Brasília, 2001.

. Ministério da Saúde. Política nacional de humanização: Humaniza SUS. Brasília, 2005. 
. Ministério da Saúde. Portaria n. 2841, de 20 de setembro de 2010. Institui, no âmbito do Sistema Único de Saúde - SUS -, o Centro de Atenção Psicossocial de Álcool e outras Drogas - 24 horas - CAPS AD III. Brasília, 2010a.

. Ministério da Saúde. Saúde mental e direitos humanos: contribuições para a IV Conferência Nacional de Saúde Mental: Intersetorial. Brasília, 2010b.

- Ministério da Saúde. Secretaria de Atenção à Saúde. Departamento de Ações Programáticas Estratégicas. Abordagens terapêuticas a usuários de cocaína/crack no Sistema Único de Saúde. Brasília, 2010c.

Ministério da Saúde. Portaria n. 336/GM, de 19 de fevereiro de 2002. Define e estabelece diretrizes para o funcionamento dos Centros de Atenção Psicossocial. Brasília, DF, 2002.

CONSELHO FEDERAL DE MEDICINA. Diretrizes gerais médicas para assistência integral ao dependente do uso do crack. Brasília, 2011.

DUARTE, M. L. C. Familiares dos usuários de crack em um CAPS AD III: avaliação das necessidades de cuidados. 2013. Tese (Doutorado em Enfermagem) - Escola de Enfermagem, Universidade Federal do Rio Grande do Sul, Porto Alegre, 2013.

DUARTE, M. L. C.; VIANA, K. R.; OLSCHOWSKY, A. Avaliação de usuários de crack sobre os grupos de familiares no centro de atenção psicossocial. Cogitare Enferm., Curitiba, v. 20, n. 1, p. 81-88, jan./mar. 2015.

FERTIG A. et al. Mulheres usuárias de crack: conhecendo suas histórias de vida. Esc. Anna Nery Rev. Enferm., v. 20, n. 2, p. 310-316, 2016.

INSTITUTO BRASILEIRO DE GEOGRAFIA E ESTATÍSTICA. IBGE. Censo 2009: estimativas das populações residentes, em $1^{\circ}$ de julho de 2009, segundo os municípios. Rio de Janeiro, 2009. Disponível em: <http://www.ibge.gov.br/home/estatistica/populacao/estimativa2009/POP2009_DOU.pdf>. Acesso em: 2 nov. 2010.

KANTORSKI, L. P. et al. Avaliação qualitativa de ambiência num Centro de Atenção Psicossocial. Ciênc. Saúde Coletiva, Rio de Janeiro, v. 16, n. 4, p. 2.059-2.066, abr. 2011.

KEMPER M. L. C. et al. Integralidade e redes de cuidado: uma experiência do PET-Saúde/Rede de Atenção Psicossocial. Interface, v. 19, n. 1, p. 995-1.003, 2015.

MINAYO, M. C.S. O desafio do conhecimento: pesquisa qualitativa em saúde. 13. ed. São Paulo: Hucitec, 2013.

NASCIMENTO, C. C.; PÍTIA, A. C. A. Oficina de trabalho corporal: uma estratégia de reabilitação psicossocial no trabalho em saúde mental. Ciênc. Cuid. Saúde, Maringá, v. 9, n. 3, p. 610-617, 2010.

OLIVEIRA, L. G.; NAPPO, S. A. Caracterização da cultura de crack na cidade de São Paulo: padrões de uso controlado. Rev. Saúde Pública, São Paulo, v. 42, n. 4, p. 664-671, 2008.

OLIVEIRA, L. M. A. C. et al. O acolhimento de familiares de pacientes internados em UTI: a tecnologia de grupo como estratégia para o cuidado de enfermagem. Rev. Esc. Enferm., USP, São Paulo, v. 44, n. 2, p. 429-36, 2010.

RIBEIRO, V. V. et al. Grupo terapêutico em fonoaudiologia: revisão de literatura. Rev. Cefac, São Paulo, v. 14, n. 3, p. 544-552, 2012.

URUGUAIANA. Secretaria Municipal de Saúde. Projeto de Implantação do CAPS AD III. Uruguaiana, 2010.

VARELA, D. S. S et al. Rede de saúde no atendimento ao usuário de álcool, crack e outras drogas. Esc. Anna Nery, v. 20, n. 2, p. 296302, 2016. 